\title{
Integrated-optics 3-way beam combiner for IOTA
}

Jean-Philippe Berger, Pierre Haguenauer, Pierre Y. Kern, Karine Rousselet-Perraut, Fabien Malbet, et al.

Jean-Philippe Berger, Pierre Haguenauer, Pierre Y. Kern, Karine RousseletPerraut, Fabien Malbet, Stephane Gluck, Laure Lagny, Isabelle SchanenDuport, Emmanuel Laurent, Alain Delboulbe, Eric Tatulli, Wesley A. Traub, Nathaniel Carleton, Rafael Millan-Gabet, John D. Monnier, Ettore Pedretti, S. Ragland, "Integrated-optics 3-way beam combiner for IOTA," Proc. SPIE 4838, Interferometry for Optical Astronomy II, (21 February 2003); doi: $10.1117 / 12.457983$

SPIE Event: Astronomical Telescopes and Instrumentation, 2002, Waikoloa, Hawai'i, United States 


\title{
An integrated-optics 3-way beam combiner for IOTA
}

\author{
J.P. Berger ${ }^{a, b}$ and P. Haguenauer ${ }^{b}$ and P. Kern ${ }^{b}$ and K. Perraut ${ }^{b}$ and F. Malbet ${ }^{b}$ \\ and S. Gluck ${ }^{b}$ and L. Lagny ${ }^{b}$ and I. Schanen ${ }^{b}$ and E. Laurent ${ }^{b}$ \\ and A. Delboulbe ${ }^{b}$ and and E. Tatulli ${ }^{b}$ \\ and W. Traub ${ }^{a}$ and N. Carleton ${ }^{a}$ and R. Millan-Gabet ${ }^{a}$ and J.D. Monnier ${ }^{a}$ \\ and E. Pedretti ${ }^{a}$ and S. Ragland ${ }^{a}$ \\ ${ }^{a}$ Harvard-Smithsonian Center for Astrophysics, 60 Garden street, Cambridge Ma, 02138 \\ ${ }^{b}$ Laboratoire d'Astrophysique de l'Observatoire de Grenoble, BP53, Grenoble Cedex-9, France \\ ${ }^{c}$ IMEP, 23 av des Martyrs, Grenoble, France
}

\begin{abstract}
We report the here the first visibility and closure-phase measurements done with the IONIC instrument at the IOTA interferometer. The IONIC instrument is presented and preliminary analysis of the results discussed. Future improvements of IONIC are envisioned.
\end{abstract}

Keywords: Interferometry, Beam combination, Integrated Optics, Phase closure

\section{INTRODUCTION}

Integrated optics (IO) technologies allow to integrate in or on a substrate waveguides using micro-lythography technologies similar to the micro-electronics technologies. The use of these masking techniques allows to implement on coin-sized chips instruments able to manipulate the light. This research field is triggered by the the need for increasing data transmission speeds which relies on new fast optical networks. Numerous IO devices are being designed for that purpose.

Kern et al.(1) and Malbet et al.(2) proposed to use IO technologies for astronomical interferometry beam combination. A good beam combiner allows multiple telescopes beams to interfere with each other and to measure with accuracy the coherence of the electric field (visibility and phase). Two laboratories in Grenoble have provided the Laboratoire d'Astrophysique de Grenoble (LAOG) with their expertise in design and manufacturing of IO chips (the IONIC collaboration). Two different technologies are currently used, ion-exchange on a glass substrate (LEMO) and silica on silicon (CEA-LETI). Successful laboratory characterizations of beam combiners were carried out $(3 ; 4 ; 5)$ demonstrating the interest of IO for beam combination at different levels. IO technologies offer several advantages.

- They allow complex optical designs, by using several basic optical functions as building blocks, one can design an optical circuit that combines several beams;

- An IO combiner contains single-mode waveguides and therefore provides a natural spatial filtering which, associated with proper calibration, leads to accurate measurements;

- An IO combiner is extremely compact;

- An IO chip (without fibers) is poorly sensitive to external perturbations;

- Whatever the number of beams to combine is, the chip will be easy to manipulate with very limited alignment constraints, mainly the injection of the light in the combiners.

Further author information: (Send correspondence to J.P.B.)

J.P.B.: E-mail: berger@obs.ujf-grenoble.fr, Telephone: 04766358 33, Address: Laboratoire d'Astrophysique de l'Observatoire de Grenoble, BP53, Grenoble Cedex 9, France 
- The optical quality (dispersion, polarization) of well optimized IO combining circuits allows the measurement of high contrast broadband fringes.

A collaboration between Laboratoire d'Astrophysique de l'Observatoire de Grenoble and Harvard-Smithsonian Center for Astrophysics was setup in 2000 to demonstrate on a real sky experiment the performances of IO combiners. A first successful experience allowed us to combine two IOTA beams with two beam combiners and proved the efficiency of the concept. Accurate visibilities were measured and several star's diameter measured (6). In this paper we report the second successful experiment to combine three beams and measure visibility closure phases. In section 2 we describe the beam combiner and its interface with the IOTA interferometer. In section 3 we carry a preliminary analysis of results. Finally in section 4 we describe our current ideas to improve performances and turn IONIC into an efficient observing instrument.

\section{INSTRUMENT DESCRIPTION}

\subsection{The Integrated Optics beam combiner}

The combiner used to obtain the first closure phase fringes was developed in collaboration with CEA-LETI. It uses silica on silicon technology and provides a pairwise combination of three beams. It was operated in the astronomical $\mathrm{H}$ band (1.5-1.8 microns). No photometric calibration signals were used since the information on photometric coupling fluctuations can be retrieved from algebraic considerations on the interferometric signals. Figure 1 displays the optical circuit of an IO combiner very similar to the one used. Three input waveguides are each splitted in 2. This results in 6 waveguides which are curved and crossed to allow a pairwise interference. The interference function is provided by an asymmetric coupler. It is designed to be as achromatic as possible. The six waveguides emerge from the 3 couplers with a spacing of 200 microns. The light coming out of those six waveguides is then focused onto 6 pixels of the infrared camera.

\subsection{The Optomechanical interface}

The IONIC beam combiner interface allows to inject starlight onto three polarization maintaining fibers that are directly connected to the integrated optics chip. It can be divided into modules with different functions (see also figure 2:

1. Focalizing modules: they are made of three off-axis parabola with 4 degrees of freedom. Fibers with rotating $\mathrm{FC} / \mathrm{PC}$ connectors are plugged at the focus.

2. Alignment module: it is made of a set of beam-splitters, an alignment source, and a reference telescope to superpose the fiber image and the star image. In its current status it's use is limited by the beam-splitter surface optical quality;

3. Beam combining module: a 5 axis mount holds the beam combiner in front of the camera allowing fine positioning in front of the detector pixels;

4. Imaging module: a pair of doublets allows to image the beam combiner six outputs onto six camera pixels. The space between the doublets is used to insert a polarizer when needed or a mirror to backward illuminate the beam combiner.

Beams coming from the three telescopes are redirected towards the IONIC interface after reflexion on a beamsplitter stage. The transmitted signal is used for angle-tracking purposes. Two of the three beam-splitters are translated with piezo to provide temporal modulation. The pairwise combination scheme requires to modulate one of the beams twice as fast as the other. The exact modulation function is a modified sawtooth designed to smooth abrupt changes susceptible to generate oscillations. 


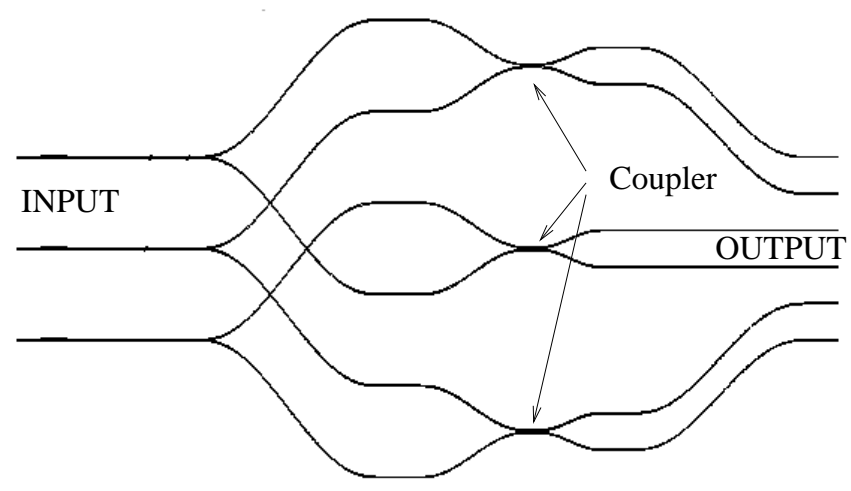

Figure 1. Description of the IONIC-IOTA three-way beam combiner. Three inputs are splitted with three "Y" junctions to provide a pairwise beam combination with another set of three couplers

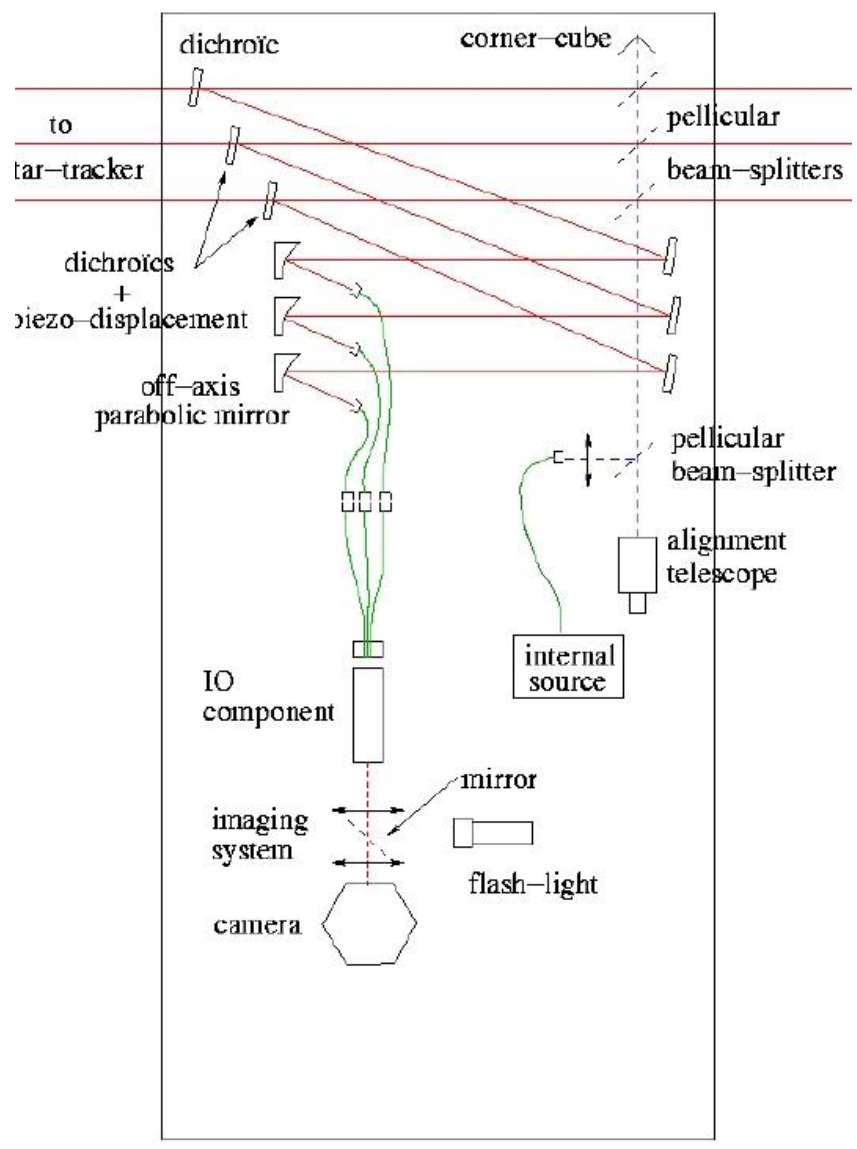

Figure 2. Schematic description of the IONIC bench (see text for details) 


\subsection{Alignment procedures.}

The alignment procedure of the beam combiner with the interferometer is standard and much alike the Fluor one.

- Focalizing the fiber at the parabola focus;

- Alignment of the interferometer with the star-tracker CCD;

- Alignment of the backwards illuminated fibers with the star tracker CCD;

- Alignment of the six outputs with the PICNIC camera ;

- Rotation of the fibers polarization axis along the same direction;

At the end of the alignment procedure the six outputs are imaged onto the PICNIC camera with almost $80 \%$ of their respective flux concentrated onto one pixel (see figure 3. A last step consist in optimizing starlight flux on the PICNIC detector by slightly moving the fiber at the focus of the parabola (this does not destroy focusing quality).

\section{PRELIMINARY RESULTS}

\subsection{An observational sequence.}

Once the interface properly aligned the next step consist in looking for the fringes in one of the baselines. The IOTA observation software allows to look for fringes simultaneously on all the baselines. Once found, a fringe group-delay tracking algorithm allows to center them at the piezo's mid-course. The following recording sequence is then started.

1. Simultaneous fringe recording on the three baselines. Typical numbers are 256 points per piezo scan with an average integration time one millisecond per point. The number of interferograms (batch) is typically 100 .

2. Shutters on beams B and C closed, flux from telescope A recorded

3. Shutters on beams A and C closed, flux from telescope B recorded

4. Shutters on beams A and B closed, flux from telescope C recorded

5. All shutters closed.

Such a sequence is recorded for the target star and interleaved as close in time as possible with a similar recording on a calibrator star. The faintest star for which we recorded data was 12 Boo $(H \approx 3.5)$

Typical time separation between those two sequences is between 5 to 10 minutes. Figure 4 displays 3 raw interferograms corresponding to the three baselines (only one output per coupler is represented here). The shutter data recorded allows to compute the photometric matrix (similar to the $\kappa$ matrix mentioned by Coudé du Foresto et al.(7)) that will be used to calibrate the photometry. 


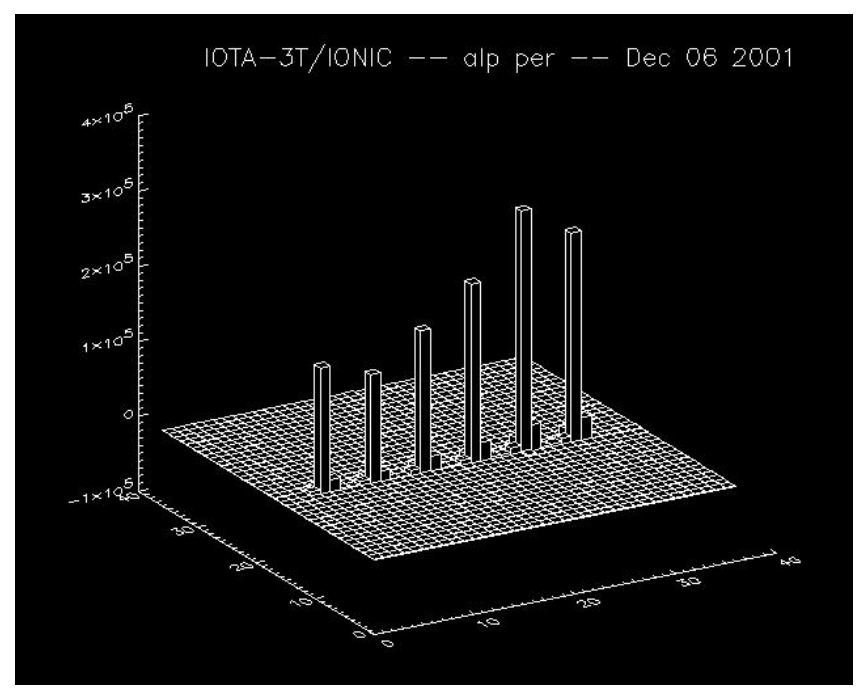

Figure 3. Image of the six beam combiner outputs on the PICNIC camera (real starlight).
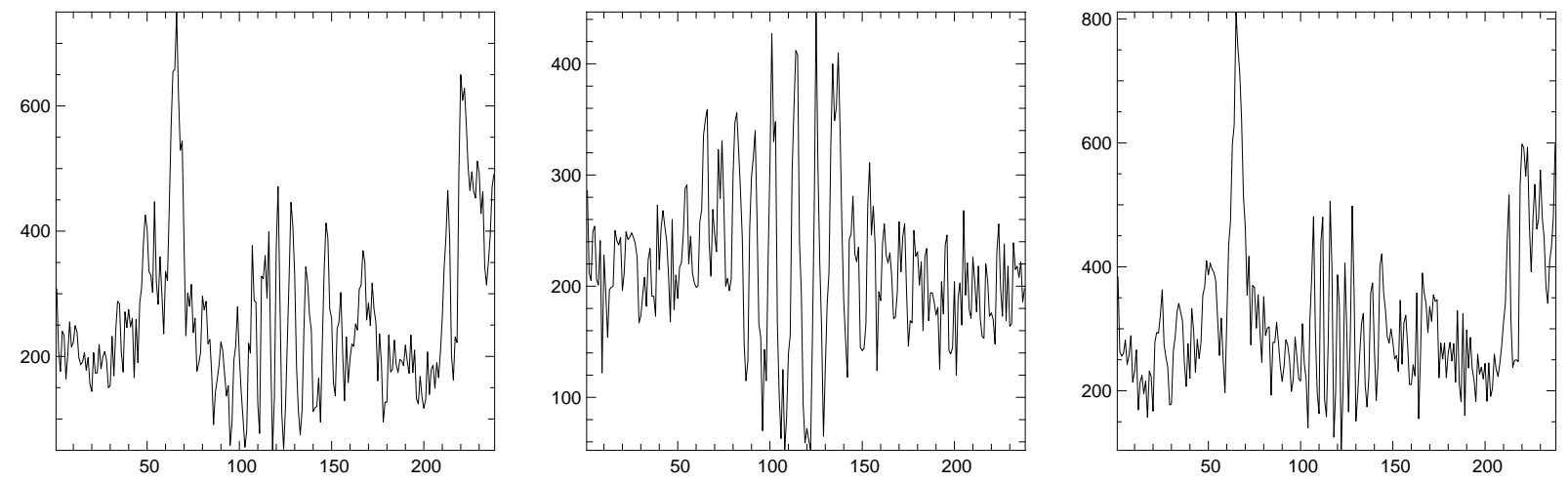

Figure 4. Three examples of raw interferograms one for each coupler output. The complementary interferogram recorded on the other side of each coupler is not represented here.
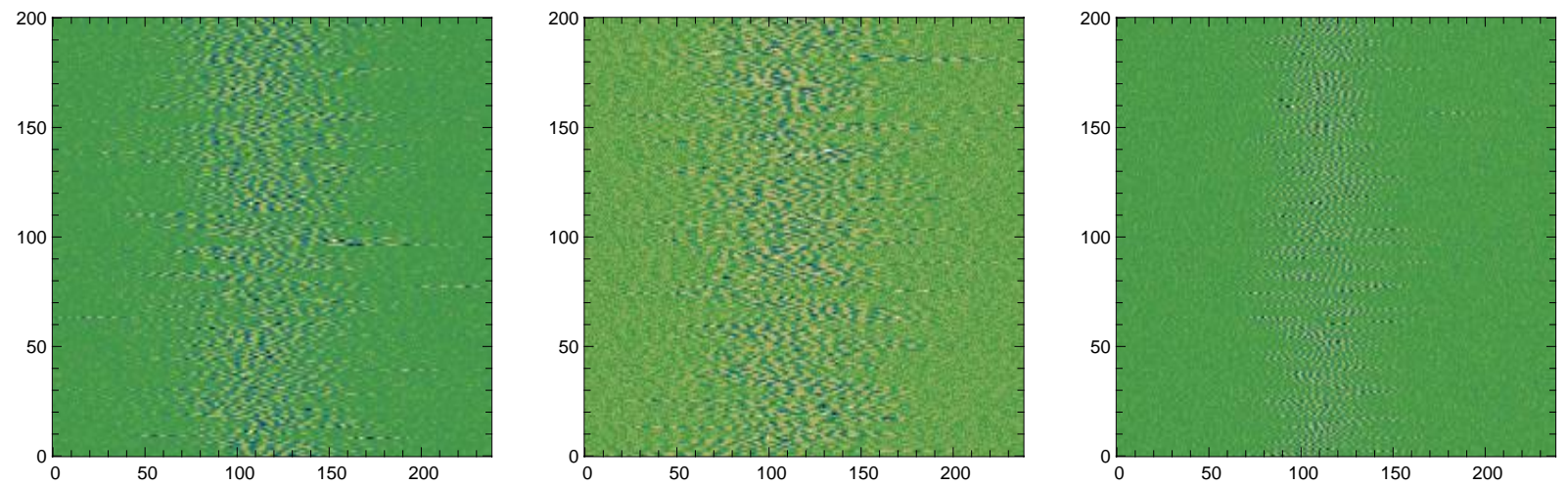

Figure 5. A cascade view of all the interferograms contained in one single batch. The three figures correspond respectively to a different coupler. The same temporal window are used in the three batches to compute closure phases in time intervals smaller than atmospheric timescales (few ms). 


\subsection{Visibility measurements.}

In order to increase instrumental contrast and to reduce polarization instability effects that occur inside the fibers observations were made using a polarizer at the output of the beam combiner.

Visibility estimation follows steps similar to what was done previously (6): the coherent flux is estimated in the Fourier space. Prior to this step the interferograms are corrected from photometry fluctuations using an algorithm initially suggested by N. Carleton and other members from the IOTA collaboration. It relies on the fact that the pairwise combination allows estimation of the three unknown coupling efficiencies using algebraic relations between the three ouptuts and the independant measurement of the photometric matrix. Moreover we note that even without such a step the good 50/50 percent balance of the couplers allows to subtract each interferogram from its $\pi$ shifted counterpart and consequently eliminate the photometry fluctuations. With the polarizer at the output we estimate the instrumental contrast to be higher to $70 \%$ although more detailed analysis are under study. Thanks to the combination of spatial filtering of the signal associated with the photometric calibration average visibility accuracy is $\leq 1 \%$.

\subsection{Phase-closure measurements}
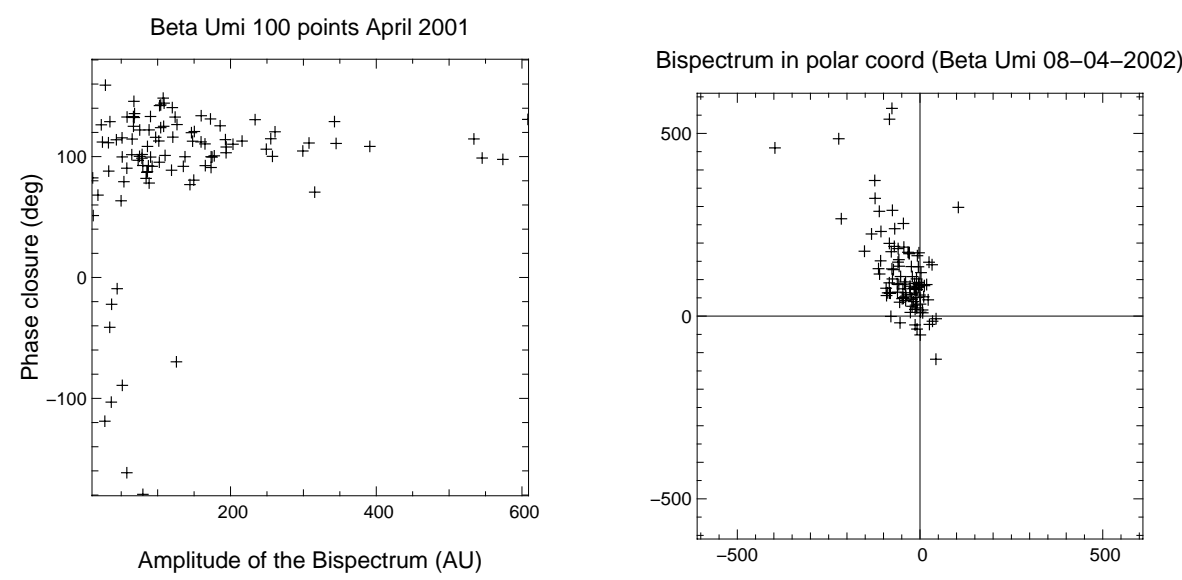

Figure 6. Two different representation of bispectrum data. These figures correspond to bispectrum measurements on a calibrator. Left Closure Phase vs. Amplitude representation, right Polar representation. Both displays show a global non-zero closure phase.

The estimation of the closure phases requires to sample the signal in a time window sufficiently narrow to freeze the atmospheric turbulence. In the current state of our data recutionn code here is how the closure-phases are computed.

For each interferogram

1. each of the 6 interferograms is cut into sub-window of time duration smaller than atmospheric timescales;

2. estimation of the bispectrum using a triplet of interferograms (output 1,3,5 and 2,4,6) for each subwindow;

3. each subwindow sampling provides one bispectrum estimation;

4. bispectrum is averaged over one set of 6 interferograms

A final bootstrapped estimation of the bispectrum is carried over the whole interferogram batch and leads to the closure phase estimation

Figure 6 displays two different representation of the same set of phase closure data (one batch) obtained on a calibrator star. Left side is a Closure Phase vs. Amplitude representation while right side is a Polar 
representation of bispectrum data. It can be clearly seen a convergence (towards $\approx 119 \pm 1.3^{\circ}$ degrees) whith increasing signal to noise (bispectrum amplitude). This value is significantly different from zero but we estimate from indirect considerations that there is no modulo correction to it. We attribute this non-zero closure-phase to the different optical paths inside the beam combiner.

\section{CONCLUSION AND FUTURE PROSPECTS.}

The current IONIC 3-way beam combiner has allowed to record first closure-phase data with the newly upgraded IOTA interferometer. Validation of the whole instrument is under way but several points are already worth mentioning:

- the alignment of the beam combiner is simple;

- the combining scheme allows to calibrate from photometry without photometric channels;

- provided that polarization is controlled the instrumental contrast level is high and average visibility accuracy on bright sources is better than $1 \%$;

- closure phase estimation shows a non-zero value for calibration stars;

For some first scientific results the reader is referred to the IOTA website:

http: //cfa-www.harvard. edu/cfa/oir/IOTA

We are now entering a period were we will improve the sensitivity and measurements accuracy. Having to polarize the signal is a constrain which comes from the highly birefringent fibers inherent behaviour. We are envisioning different options to overcome these difficulties:

- Reduce fiber instability by insulating them from thermal effects and mechanical contrains.

- Use a polarization splitter at the output and record interferograms from both polarizations. This requires that the IO combiner+fiber association to be optimized for limited crosstalk between polarizations;

A first alternative would be to try to modify birefringence by stressing and inducing torques to the PM fibers (e.g. using Lefevre loops). Another possibility would be inject direcly starlight into the integrated optics beam combiner which is much more stable than the fibers.

Once these effect will be mastered we plan to focus our efforts in studing more subtle effects such as the influence of the combiner chromaticity and differential birefringence on the closure-phases.

\section{ACKNOWLEDGMENTS}

The integrated optics research program at LAOG is funded by CNES and the CNRS ASHRA program. IOTA is operated by the Smithsonian Institution. JPB acknowledges financial support from JPL through a Michelson Postdoctoral fellowship. We wish to thank all the members of the IONIC collaboration for their strong involvement in the project. We particularly thank Pierre Labeye for fruitful discussions. 


\section{References}

[1] P. Kern, F. Malbet, I. Schanen-Duport, and P. Benech, "Integrated optics single-mode interferometric beam combiner for near-infrared astronomy," in Astrofib' 96: Integrated optics for astronomical interferometry, F. Kern, P. Malbet, ed., (Grenoble), 1996.

[2] F. Malbet, P. Kern, I. Schanen-Duport, J.-P. Berger, K. Rousselet-Perraut, and P. Benech, "Integrated optics for astronomical interferometry. I. Concept and astronomical applications," Astronomy and Astrophysics, Supplement 138, pp. 135-145, July 1999.

[3] J. P. Berger, K. Rousselet-Perraut, P. Kern, F. Malbet, I. Schanen-Duport, F. Reynaud, P. Haguenauer, and P. Benech, "Integrated optics for astronomical interferometry. II. First laboratory white-light interferograms," Astronomy and Astrophysics, Supplement 139, pp. 173-177, Oct. 1999.

[4] P. Haguenauer, J. P. Berger, K. Rousselet-Perraut, P. Kern, F. Malbet, and I. Schanen-Duport, "Integrated optics for astronomical interferometry. II. First laboratory white-light interferograms," Applied Optics 39, pp. 2130-2139, May 2000.

[5] P. Haguenauer, M. Severi, I. Schanen, K. Perraut, J. Berger, Y. Duchene, M. Lacolle, P. Kern, F. Malbet, and P. Benech, "Planar optics three-telescope beam combiners for astronomical interferometry.," in Proc. SPIE, Interferometry in Optical Astronomy, Munich, pp. 1107-1115, jun 2000.

[6] J. P. Berger, P. Haguenauer, P. Kern, K. Perraut, F. Malbet, I. Schanen, M. Severi, R. Millan-Gabet, and W. Traub, "Integrated optics for astronomical interferometry: VI first measurements of stars.," å 3, pp. L31-L34, sep 2001.

[7] V. Coude Du Foresto, S. Ridgway, and J.-M. Mariotti, "Deriving object visibilities from interferograms obtained with a fiber stellar interferometer," Astronomy and Astrophysics, Supplement 121, pp. 379-392, Feb. 1997. 